\title{
Diabetic ketoacidosis associated with outpatient treatment using continuous so subcutaneous insulin infusion
}

\author{
A. J. M. Boulton \\ M.B., M.R.C.P. \\ J. DRURY \\ M.B., M.R.C.Path.
}

\author{
G. KNIGHT \\ M.B., M.R.C.P. \\ J. D. WARD \\ M.D., F.R.C.P.
}

Royal Hallamshire Hospital, Sheffield S10 2JF

\begin{abstract}
Summary
The occurrence of ketoacidosis in out-patient diabetics treated with continuous subcutaneous insulin infusion (CSII) has received little attention. We report two such cases of ketosis, each precipitated by infection and occurring in patients previously well controlled on CSII. This report illustrates that the risk of ketoacidosis is ever present in insulin-treated patients, whether the insulin is infused by pump or injected intermittently.
\end{abstract}

KEY WORDS: diabetes mellitus, insulin therapy, abscesses.

\section{Introduction}

Recent studies have confirmed that continuous subcutaneous insulin infusion (CSII) can lead to near normalization of blood glucose levels in out-patient diabetics (Pickup et al., 1980; Boulton et al., 1982; Schiffren and Belmonte, 1982). Although there have been several reports of hypoglycaemic reactions in diabetics treated by CSII as out-patients (Lock and Rigg, 1981; Boulton et al., 1982; Mecklenburg et al., 1982; Schiffren and Belmonte, 1982), ketoacidosis has received little attention. We have recently started a feasibility study into the use of CSII in a diabetic clinic (Knight et al., 1982), and here report two cases of ketoacidosis in type one (insulin-dependent) diabetics treated with CSII, the first of which was precipitated by an infection at the infusion site.

\section{Case reports}

Case 1

A 46-year-old woman had started CSII 17 months ago as part of a study of strict glucoregulation in diabetic neuropathy (Boulton et al., 1982). Three days before this admission, she developed an abscess at the infusion site on the anterior abdominal wall and was treated with oral ampicillin and cloxacilling. by her general practitioner. Despite changing the infusion site, 2 days later she complained of thirste and polyuria and adjusted her insulin infusion rate according to the blood sugar levels carried out bj home monitoring. Her capillary blood glucose at $10 \mathrm{O}$ pm was $7.0 \mathrm{mmol} /$ litre according to her reading (BMC stick, Boehringer Ingelheim), but she awoke at 4 amf the next morning with nausea, thirst and vomiting She immediately contacted the hospital and receivet instructions to come straight to the ward for admoss sion. The plasma glucose on admission to hospitit was $26.3 \mathrm{mmol} /$ litre and the bicarbonate was $\mathrm{mmol} /$ litre. She was treated with intravenous fluidds and intramuscular insulin and recovered rapidly. The abscess required drainage under local anaesthesion and pus taken from this site grew Staphylococcus aureus. Swabs taken from the anterior nares, axilla, $\overrightarrow{\vec{\rho}}$ umbilicus and groin did not grow this organism. She was discharged home on twice daily injections in view of the previous skin infections at the infusion. site.

\section{Case 2}

A 47-year-old man had started CSII 2 weekso before his admission, as part of a long-term feasibility? study into the use and acceptability of CSII (Knigho et al., 1982). Diabetic control was satisfactory on the day before admission with capillary blood glucoses readings between 4 and $10 \mathrm{mmol} /$ litre (BM sticks) N At night he awoke after an hour's sleep with severe nausea and vomited profusely during the rest of the night. He telephoned the unit the next day and was advised to come to the hospital for treatment. On admission to hospital he was dehydrated althoughe the infusion pump was functioning and there was noo evidence of skin infection. Blood sugar was 750 
$\mathrm{mmol} /$ litre, plasma bicarbonate $9.0 \mathrm{mmol} /$ litre and urea $16.7 \mathrm{mmol} /$ litre. He was treated with intramuscular insulin and intravenous fluids and recovered rapidly although he developed symptoms and signs of an upper respiratory tract infection on the second day of admission. Blood cultures taken at this time were negative. The patient elected to continue with CSII as he preferred this to conventional insulin therapy and he was discharged home. It was presumed that his ketosis was precipitated by the mild upper respiratory tract infection.

\section{Discussion}

In both cases, the episodes of ketoacidosis occurred in patients previously well controlled with CSII, though in neither case was there any evidence of pump malfunction. Despite earlier reports to the contrary (Pickup et al., 1980), skin infections at the infusion site represent a potential problem during CSII (Pietri and Raskin, 1981) and this is an indication for immediate resiting of the infusion set. However, this precaution and the prescription of antibiotics did not prevent the development of a staphylococcal abscess which precipitated ketosis in our first patient. Despite this patient's preference for CSII, it was decided that the risk of further serious skin infections precluded the continuation of this form of insulin delivery. Although the recent report from Seattle on the clinical use of CSII in 100 patients described cutaneous infection in $10 \%$ of patients, these all responded to antibiotic therapy (Mecklenburg et al., 1982).

It has recently been reported that deliberate cessation of CSII for $9 \mathrm{hr}$ did not lead to any dangerous clinical deterioration (Pickup et al., 1982). However, in the presence of infection and despite increased insulin dosage in our first case, our patients rapidly developed ketosis over a few hours. Intercurrent illnesses have previously been reported in earlier long-term studies of CSII, and these have been controlled by increasing the rate of insulin infusion (Boulton et al., 1982; Schiffren and Belmonte, 1982). The present report illustrates that the risk of ketoacidosis is ever present in insulin treated patients, whether the insulin be infused or injected intermittently. Self-monitoring of urinary ketones may have led to earlier recognition of the ketosis by the patients. However, if either patient had not been able to contact our unit directly, the outcome may well have been extremely serious.

We feel that CSII should only be used in centres where full cover and expertise can be offered, and we would therefore suggest the following as minimum requirements for hospitals treating patients with CSII:

(1) Adequate education of patients before starting CSII on the techniques and possible problems. Each patient should be given an illustrated instruction manual.

(2) Other members of the diabetic unit, including qualified nursing staff and junior medical staff, should be familiar with CSII.

(3) 24-hr hospital cover by staff experienced in CSII should be available: patients should be encouraged to contact the unit as soon as problems, however trivial, arise. Many minor problems may be solved by telephone consultation.

The recent report from the U.S.A. of several deaths in diabetics on CSII is further evidence of the care needed with this new technique (Anonymous, 1982). It would be unfortunate if a treatment of potentially great benefit to a large number of diabetics were discredited because the utmost attention were not provided during the early days of its usage. We conclude that ketosis during CSII is a recognized risk and that it may develop rapidly.

\section{References}

ANONYMOUS (1982) Deaths in diabetics using insulin infusion pumps. Lancet, i, 636.

Boulton, A.J.M., Drury, J., Clarke, B. \& Ward, J.D. (1982) Continuous subcutaneous insulin infusion in the management of painful diabetic neuropathy. Diabetes Care, 5, 386.

KNIGHT, G., Boulton, A.J.M., DruRy, J., Moses, J.L., Bradley, C. \& WARD, J.D. (1982) Initial report of a feasibility study of the introduction of continuous subcutaneous insulin infusion in a diabetic clinic. Diabetologia, 23, 470.

Lock, D.R. \& RIGG, L.A. (1981) Hypoglycaemic coma associated with subcutaneous insulin infusion by portable pump. Diabetes Care, 4, 389.

MeCKlenburg, R.S., Benson, J.W., BeckeR, N.M., BRAZel, P.L., Fredlund, P.N., Metz, R.J., Nielsen, R.L., Sannar, C.A. \& STEENROD, W.J. (1982) Clinical use of the insulin infusion pump in 100 patients with Type I diabetes. New England Journal of Medicine, 307, 513.

Pickup, J.C., Keen, H., Viberti, G.C., White, M.C., Kohner, E.M., Parsons, J.A. \& AlberTi, K.G.M.M. (1980) Continuous subcutaneous insulin infusion in the treatment of diabetes mellitus. Diabetes Care, 3, 290.

Pickup, J.C., Viberti, G.C., Bilous, R.W., Keen, H., Alberti, K.G.M.M., HOME, P.D. \& BiNDER, C. (1982) Safety of continuous subcutaneous insulin infusion: metabolic deterioration and glycaemic auto-regulation after deliberate cessation of infusion. Diabetologia, 22, 175.

PIETRI, A. \& RASKIN, P. (1981) Cutaneous complications of chronic subcutaneous insulin infusion therapy. Diabetes Care, 4, 624.

SCHIFfREN, A. \& BelmonTE, M.M. (1982) Comparison between continuous subcutaneous insulin infusion and multiple injections of insulin. Diabetes, 31, 255. 